\title{
Classes and theories of trees associated with a class of linear orders
}

\author{
Valentin Goranko ${ }^{1}$ and Ruaan Kellerman ${ }^{2, *}$ \\ ${ }^{1}$ Department of Informatics and Mathematical Modelling, Technical \\ University of Denmark, Lyngby, Denmark and ${ }^{2}$ Department of Mathematics \\ and Applied Mathematics, University of Pretoria, Pretoria, South Africa
}

\begin{abstract}
Given a class of linear order types $\mathcal{C}$, we identify and study several different classes of trees, naturally associated with $\mathcal{C}$ in terms of how the paths in those trees are related to the order types belonging to $\mathcal{C}$. We investigate and completely determine the set-theoretic relationships between these classes of trees and between their corresponding first-order theories. We then obtain some general results about the axiomatization of the first-order theories of some of these classes of trees in terms of the first-order theory of the generating class $\mathcal{C}$, and indicate the problems obstructing such general results for the other classes. These problems arise from the possible existence of nondefinable paths in trees, that need not satisfy the first-order theory of $\mathcal{C}$, so we have started analysing first-order definable and undefinable paths in trees.
\end{abstract}

Keywords: Trees, linear orders, first-order theories, definable and non-definable paths.

\section{Introduction}

Trees occur naturally in many mathematical settings as important partial orders. Every tree is naturally associated with a set of linear orders representing its paths. Conversely, with every set $\mathcal{C}$ of linear order types one can associate various classes of trees, the paths of which belong, or are related, to $\mathcal{C}$. Trees are far more complex structures than linear orders, and it is interesting and important to analyse the structural and logical relationships between trees and their sets of paths. While much is known about some important classes of trees and their first-order theories, such as finite trees [1], well-founded trees [2], and finitely branching trees $[4,7]$, the only systematic study so far, of which we are aware, of classes of trees that naturally arise from a given set of linear order types is [5], in the context of temporal logics. The objective of this article is to continue and extend that line of research by investigating the variety of classes of trees, the paths in which are naturally associated with a given class of linear orders and of the first-order theories of these classes. In a subsequent work, we apply present results to obtain complete axiomatizations of the first-order theories of some important classes of trees.

The article is structured as follows: after a preliminary Section 2, we introduce in Section 3 several classes of trees associated in terms of the paths in those trees with a given class of linear orders. Then, in Section 4 we analyse and completely describe the set-theoretic

\footnotetext{
*E-mail: ruaan.kellerman@up.ac.za
}

Vol. 19 No. 1, (C) The Author 2010. Published by Oxford University Press. All rights reserved. 
relationships between these classes of trees, whereas in Section 5 we completely describe the relationships between their respective first-order theories. In Section 6, we discuss the problem of axiomatizing these theories given a first-order axiomatization of the generating class of linear orders. In Section 7, we take a closer look at the different types of paths emerging in trees associated with a given class of linear orders and how they are related with the first-order properties of such trees. We end with brief concluding remarks summarizing the article and discussing the arising open problems and further research directions.

\section{Preliminaries}

Let $\mathfrak{A}$ be a structure and let $\varphi\left(x_{1}, \ldots, x_{n}\right)$ be a first-order formula. The domain of $\mathfrak{A}$ is denoted as $|\mathfrak{A}|$ or simply as $A$. Let $c_{1}, \ldots, c_{n} \in|\mathfrak{A}|$ and put $\bar{x}=\left(x_{1}, \ldots, x_{n}\right)$ and $\bar{c}=\left(c_{1}, \ldots, c_{n}\right)$. Then $\varphi\left(x_{1}, \ldots, x_{n}\right)$ is also written as $\varphi(\bar{x})$. When evaluating the truth of $\varphi$ in $\mathfrak{A}$ when the elements $c_{i}$ are substituted for $x_{i}$ for every $i(1 \leqslant i \leqslant n)$, we also denote the expression $\mathfrak{A} \models \varphi\left(c_{1} / x_{1}, \ldots, c_{n} / x_{n}\right)$ as $\mathfrak{A} \models \varphi(\bar{c} / \bar{x})$. When enriching the signature of $\mathfrak{A}$ with $c_{1}, \ldots, c_{n}$ as parameters, we also denote $\left(\mathfrak{A} ; c_{1}, \ldots, c_{n}\right)$ as $(\mathfrak{A} ; \bar{c})$ and $(\mathfrak{A} ; \bar{c}) \models \varphi\left(c_{1}, \ldots, c_{n}\right)$ as $(\mathfrak{A} ; \bar{c}) \models \varphi(\bar{c})$.

For $\bar{a}=\left(a_{1}, \ldots, a_{k}\right)$ and $\bar{b}=\left(b_{1}, \ldots, b_{n}\right)$, the notation $\bar{a} c$ indicates the $(k+1)$-tuple $\left(a_{1}, \ldots, a_{k}, c\right)$ and $\bar{a} \bar{b}$ indicates the $(k+n)$-tuple $\left(a_{1}, \ldots, a_{k}, b_{1}, \ldots, b_{n}\right)$.

The quantifier rank of a formula $\varphi$ is denoted as $\operatorname{qr}(\varphi)$. Elementary equivalence between structures is denoted by $\equiv$ and $n$-equivalence (equivalence with respect to all sentences of quantifier rank at most $n$ ) by $\equiv_{n}$.

\subsection{Relativizing first-order formulas}

Relativizations give a neat method for imposing first-order properties on definable substructures of a structure. The following definition and results are taken from [6, pp. 259-260].

Let $\mathfrak{A}$ be any structure and let $a_{1}, \ldots, a_{k} \in|A|$. Fix $\bar{x}=\left(x_{1}, \ldots, x_{n}\right), \bar{y}=\left(y_{1}, \ldots, y_{k}\right)$ and $\bar{a}=$ $\left(a_{1}, \ldots, a_{k}\right)$.

Definition $1[6]$

Let $\varphi(\bar{x})$ and $\theta(u, \bar{y})$ be any first-order formulas. The relativization of $\varphi$ to $\theta$, denoted $\varphi^{\theta}$ (where $\varphi^{\theta}=\varphi^{\theta}(\bar{x}, \bar{y})$ ), is defined as follows:

(i) if $\varphi$ is atomic then $\varphi^{\theta}:=\varphi$;

(ii) if $\varphi=\neg \psi$ then $\varphi^{\theta}:=\neg\left(\psi^{\theta}\right)$;

(iii) if $\varphi=\psi_{1} \star \psi_{2}$ then $\varphi^{\theta}:=\psi_{1}^{\theta} \star \psi_{2}^{\theta}$, where $\star$ is any of $\vee, \wedge, \rightarrow$ or $\leftrightarrow$;

(iv) if $\varphi=\exists x \psi$ then $\varphi^{\theta}:=\exists x\left(\theta(x, \bar{y}) \wedge \psi^{\theta}\right)$; and

(v) if $\varphi=\forall x \psi$ then $\varphi^{\theta}:=\forall x\left(\theta(x, \bar{y}) \rightarrow \psi^{\theta}\right)$.

Note that if $\varphi$ is quantifier free then $\varphi^{\theta}$ contains the variables $y_{1}, \ldots, y_{k}$ vacuously, while if $\varphi$ contains quantifiers then the variables $y_{1}, \ldots, y_{k}$ will appear explicitly in $\varphi^{\theta}$.

Define

$$
(\mathfrak{A} ; \bar{a})^{\theta}:=\{b \in|\mathfrak{A}|:(\mathfrak{A} ; \bar{a}) \mid=\theta(b / u, \bar{a})\}
$$


Proposition 2 [6]

Let $\varphi(\bar{x})$ and $\theta(u, \bar{y})$ be any first-order formulas. For any $b_{1}, \ldots, b_{n} \in(\mathfrak{A} ; \bar{a})^{\theta}$ and with $\bar{b}=$ $\left(b_{1}, \ldots, b_{n}\right)$,

$$
\mathfrak{A} \models \varphi^{\theta}(\bar{b} / \bar{x}, \bar{a} / \bar{y}) \Leftrightarrow(\mathfrak{A} ; \bar{a})^{\theta} \models \varphi(\bar{b} / \bar{x}) .
$$

Corollary $3[6]$

Let $\mathfrak{A}$ be a structure, let $\sigma$ be a first-order sentence and let $\theta(u, \bar{y})$ be a first-order formula. Then

$$
\mathfrak{A} \models \sigma^{\theta}(\bar{a} / \bar{y}) \Leftrightarrow(\mathfrak{A} ; \bar{a})^{\theta} \models \sigma .
$$

Corollary $4[6]$

Let $\mathfrak{A}$ be a structure, let $\sigma$ be a first-order sentence and let $\theta(u)$ be a first-order formula. Then

$$
\mathfrak{A} \models \sigma^{\theta} \Leftrightarrow \mathfrak{A}^{\theta}=\sigma .
$$

\subsection{Characteristic formulas}

Characteristic formulas give a syntactic formalization of the Ehrenfeucht-Fraïssé game played on a pair of structures. The following definition and results are borrowed from [2]. An excellent account of characteristic formulas can also be found in [3].

Fix structures $\mathfrak{A}$ and $\mathfrak{B}$. Let $\bar{a}=\left(a_{1}, \ldots, a_{k}\right)$ and $\bar{b}=\left(b_{1}, \ldots, b_{k}\right)$, where $a_{1}, \ldots, a_{k} \in|\mathfrak{A}|$ and $b_{1}, \ldots, b_{k} \in|\mathfrak{B}|$. Put $\bar{x}=\left(x_{1}, \ldots, x_{k}\right)$.

Definition 5 [2]

For $n \in \mathbb{N}$ we define the formula $\llbracket(\mathfrak{A} ; \bar{a}) \rrbracket^{n}$ (with $\left.\llbracket(\mathfrak{A} ; \bar{a}) \rrbracket^{n}=\llbracket(\mathfrak{A} ; \bar{a}) \rrbracket^{n}(\bar{x})\right)$ inductively as follows:

(i) $\llbracket(\mathfrak{A} ; \bar{a}) \rrbracket^{0}:=\bigwedge\{\varphi(\bar{x}): \varphi$ an atomic or negated atomic

(ii) $\llbracket(\mathfrak{A} ; \bar{a}) \rrbracket^{m+1}:=\bigwedge_{a_{k+1} \in|\mathfrak{A}|} \exists x_{k+1} \llbracket\left(\mathfrak{A} ; \bar{a} a_{k+1}\right) \rrbracket^{m} \wedge$ formula with $\mathfrak{A} \models \varphi(\bar{a} / \bar{x})\} ;$

$$
\forall x_{k+1} \bigvee_{a_{k+1} \in|\mathfrak{A}|} \llbracket\left(\mathfrak{A} ; \bar{a} a_{k+1}\right) \rrbracket^{m}
$$

The formula $\llbracket(\mathfrak{A} ; \bar{a}) \rrbracket^{n}$ is known as the $n$-characteristic of $\bar{a}$ in $\mathfrak{A}$.

The formula $\llbracket(\mathfrak{A} ; \bar{a}) \rrbracket^{n}$ describes any (the first conjunct in clause (ii) of Definition 5 ) and all (the second conjunct in clause (ii) of Definition 5) ways to relate $n$-tuples of elements from $\mathfrak{A}$ to the elements in $\bar{a}$.

LEMMA 6 [2]

(i) $\mathfrak{A} \models \llbracket(\mathfrak{A} ; \bar{a}) \rrbracket^{n}(\bar{a} / \bar{x})$; and

(ii) the formula $\llbracket(\mathfrak{A} ; \bar{a}) \rrbracket^{n}$ has quantifier rank $n$.

THEOREM $7[2]$

For $n \in \mathbb{N}$ the following conditions are equivalent:

(i) $(\mathfrak{A} ; \bar{a}) \equiv_{n}(\mathfrak{B} ; \bar{b})$;

(ii) $\mathfrak{B} \models \llbracket(\mathfrak{A} ; \bar{a}) \rrbracket^{n}(\bar{b} / \bar{x})$; and

(iii) the formulas $\llbracket(\mathfrak{A} ; \bar{a}) \rrbracket^{n}$ and $\llbracket(\mathfrak{B} ; \bar{b}) \rrbracket^{n}$ are equivalent. 


\section{Corollary 8}

Let $n \in \mathbb{N}$. The following conditions are equivalent:

(i) $\mathfrak{A} \equiv{ }_{n} \mathfrak{B}$;

(ii) $\mathfrak{B} \models \llbracket \mathfrak{A} \rrbracket^{n}$; and

(iii) the formulas $\llbracket \mathfrak{A} \rrbracket^{n}$ and $\llbracket \mathfrak{B} \rrbracket^{n}$ are equivalent.

Hence, the $n$-characteristics of empty tuples are canonical objects associated with classes of structures which are $n$-equivalent.

When working with a finite signature, there will be only finitely many $n$-characteristics of $k$-tuples.

Theorem 9 [2]

Let $\left\{\mathfrak{A}_{i}\right\}_{i \in I}$ be a class of structures over the same finite signature. Let $n, k \in \mathbb{N}$ and let $\bar{a}_{i}$ be a $k$-tuple from $\mathfrak{A}_{i}$ for every $i \in I$. The set of pairwise non-equivalent formulas $\llbracket\left(\mathfrak{A}_{i} ; \bar{a}_{i}\right) \rrbracket^{n}(i \in I)$ is finite.

\subsection{Trees}

A tree is a partially ordered set $(T ;<)$ satisfying the following two properties:

(i) for every $x \in T$ the set $\{y \in T: y<x\}$ is totally ordered, and

(ii) for every $x, y \in T$, there exists $z \in T$ such that $z \leqslant x, y$.

Thus, the class of trees can be defined in the language containing the binary relation < together with the usual equality relation $=$, using the first-order theory consisting of the following sentences:

$$
\begin{aligned}
& \mathrm{A}_{1}: \forall x(\neg x<x) ; \\
& \mathrm{A}_{2}: \forall x \forall y \forall z(x<y \wedge y<z \rightarrow x<z) ; \\
& \mathrm{A}_{3}: \forall x \forall y \forall z(y<x \wedge z<x \rightarrow(x<y \vee x=y \vee y<x)) \\
& \mathrm{A}_{4}: \forall x \forall y \exists z((z<x \vee z=x) \wedge(z<y \vee z=y))
\end{aligned}
$$

We will denote the first-order theory axiomatized with the four sentences above by Tree.

When the context allows we will simply write $T$ for $(T ;<)$. Also, we write $x \leqslant y$ as a shorthand for $x<y \vee x=y$. The elements of a tree are called nodes. If $a<b$ then $a$ is called the predecessor of $b$ and $b$ is called a successor of $a$; if there is no $c$ with $a<c<b$ then $a$ is called the immediate predecessor of $b$ and $b$ is called an immediate successor of $a$. A tree is called binary if every node in it has precisely two immediate successors. A <maximal node is called a leaf. The set of leaves can be defined using the first-order formula $\operatorname{leaf}(x):=\forall y(x \leqslant y \rightarrow x=y)$.

A maximal totally ordered set of nodes is called a path. Using Zorn's Lemma, it is easy to see that every totally ordered subset of a tree is contained in a path. For any order type $\alpha$, a path $A$ in a tree is called an $\alpha$-path if $A$ is isomorphic with $\alpha$. The binary tree in which every path is an $\alpha$-path will be denoted $B_{\alpha}$. As usual, we denote by $\omega$ the order type of the positive integers, by $\omega^{\star}$ the order type of the negative integers, and by $\zeta$ the order type of all integers.

A tree $T$ is called well-founded when every non-empty set of nodes from $T$ contains a minimal node. 
A tree is called downwards discrete when every non-root node in $T$ has an immediate predecessor. $T$ is called weakly upwards discrete when every non-leaf node has an immediate successor, and upwards discrete when, for every path $X$ in $T$, every non-leaf node in $X$ has an immediate successor belonging to $X$. T is called weakly discrete when it is both downwards discrete as well as weakly upwards discrete, and discrete when it is both downwards discrete and upwards discrete. Note that all these properties are first-order definable.

A discrete tree is called finitely branching when every node in that tree has finitely many (possibly none) immediate successors. ${ }^{1}$

Given a tree $T$ and $a \in T$, define

$$
\begin{aligned}
& a_{>}:=\{x \in T: x<a\}, \\
& a_{\geqslant}:=\{x \in T: x \leqslant a\}, \\
& a_{<}:=\{x \in T: a<x\}, \\
& a_{\leqslant}:=\{x \in T: a \leqslant x\} .
\end{aligned}
$$

The sets $a_{>}, a_{\geqslant}, a_{<}$and $a_{\leqslant}$will also be treated as substructures of $T$. The sets $a_{>}$and $a_{\geqslant}$ are always linear orders, while the set $a_{\leqslant}$is always a tree - the generated subtree of $T$ rooted at $a$.

For $k \in \mathbb{N}$ and $\varphi(x, \bar{z})$ any formula with $\bar{z}=\left(z_{1}, \ldots, z_{k}\right)$, define the formula

$$
\begin{aligned}
& \pi_{\varphi}(\bar{z}):=\exists x \varphi(x, \bar{z}) \wedge \forall x \forall y(\varphi(x, \bar{z}) \wedge \varphi(y, \bar{z}) \rightarrow(x<y \vee x=y \vee y<x)) \wedge \\
& \forall x \forall y(x<y \wedge \varphi(y, \bar{z}) \rightarrow \varphi(x, \bar{z})) \wedge \neg \exists x \forall y(\varphi(y, \bar{z}) \rightarrow y<x) .
\end{aligned}
$$

Note that, if $\varphi$ has quantifier rank $n$, then $\pi_{\varphi}$ has quantifier rank $n+2$.

The formula $\pi_{\varphi}$ formalizes the claim that the formula $\varphi$ defines a path. Verifying that is straightforward, thus obtaining the following.

Proposition 10

Let $T$ be a tree with $c_{1}, \ldots, c_{k} \in T$ and put $\bar{c}=\left(c_{1}, \ldots, c_{k}\right)$. The formula $\varphi(x, \bar{c})$ defines a path in $(T ; \bar{c})$ if and only if $T \models \pi_{\varphi}(\bar{c} / \bar{z})$.

By the proposition above, if $T_{1} \equiv T_{2}$ then a formula $\varphi$ defines a path in $T_{1}$ iff it defines a path in $T_{2}$.

A substructure $S$ of a tree $T$ which is itself a tree is called a subtree of $T . S$ is called upwards closed in $T$ when it satisfies the property that for all $x, y \in T$, whenever $x \in S$ and $x<y$ then $y \in S$.

Proposition $11[4]$

Let $T_{1}=\left(T_{1} ;<_{T_{1}}\right)$ be a tree and let $\left\{\left(S_{i} ;<_{S_{i}}\right): i \in I\right\}$ be a pairwise disjoint set of upwards closed subtrees of $T_{1}$, where the order $<_{S_{i}}$ is the order $<_{T_{1}}$ restricted to the domain $S_{i}$. For every $i \in I$, let $S_{f(i)}=\left(S_{f(i)} ;<_{S_{f(i)}}\right)$ be a tree with $S_{i} \equiv_{n} S_{f(i)}$. Let $T_{2}$ be the tree obtained from $T_{1}$ by replacing every subtree $S_{i}$ with the tree $S_{f(i)}$. Formally, we define $T_{2}=\left(T_{2} ;<_{2}\right)$ as follows:

- $\left|T_{2}\right|:=\left(T_{1} \backslash \bigcup_{i \in I} S_{i}\right) \cup \bigcup_{i \in I} S_{f(i)}$, and

\footnotetext{
${ }^{1}$ This definition of finite branching is not the most general, nor necessarily the most intuitively acceptable, but it is sufficient for the purposes of this article. For further details and discussion on finite branching see [7] and [4].
} 
- for $x, y \in T_{2}, x<_{T_{2}} y$ if and only if one of the following conditions are satisfied:

(i) $x, y \in T_{1} \backslash \bigcup_{i \in I} S_{i}$ and $x<_{T_{1}} y$, or

(ii) $x, y \in S_{f(i)}$ for some $i$ and $x<_{S_{f(i)}} y$, or

(iii) $x \in T_{1} \backslash S_{i}$ and $y \in S_{f(i)}$ for some $i$, and $x<_{T_{1}} z$ for some $z \in S_{i}$.

Then $T_{1} \equiv_{n} T_{2}$.

\section{Classes of trees associated with a given class of linear orders}

Here, we define several classes of trees naturally associated, from the viewpoint of first-order logic, with a given set of linear order types $\mathcal{C}$. We will call these classes of trees $\mathcal{C}$-classes.

DeFinition 12

A tree $T$ is called a:

(i) $\mathcal{C}$-tree when every path in $T$ is an $\alpha$-path for some $\alpha \in \mathcal{C}$;

(ii) uniformly $\mathcal{C}$-like tree $(U$-C -like tree) if $T \equiv S$ for some $\mathcal{C}$-tree $S$;

(iii) $\mathcal{C}$-like tree if, for every $n \in \mathbb{N}$, there is a $\mathcal{C}$-tree $S$ such that $T \equiv_{n} S$;

(iv) pathwise uniformly $\mathcal{C}$-like tree $(P U$-C -like tree) if, for every path $X$ in $T$, there exists $\alpha \in \mathcal{C}$ such that $X \equiv \alpha$;

(v) pathwise $\mathcal{C}$-like tree $(P-\mathcal{C}$-like tree) if, for every path $X$ in $T$ and for every $n \in \mathbb{N}$, there exists $\alpha \in \mathcal{C}$ such that $X \equiv_{n} \alpha$;

(vi) definably $\mathcal{C}$-tree $(D$-C -tree $)$ if every parametrically definable path $X$ in $T$ is an $\alpha$-path for some $\alpha \in \mathcal{C}$ (dependent on $X$ );

(vii) definably uniformly $\mathcal{C}$-like tree $(D U$-C -like tree) if, for every parametrically definable path $X$ in $T$, there exists $\alpha \in \mathcal{C}$ such that $X \equiv \alpha$;

(viii) definably $\mathcal{C}$-like tree $(D$-C -like tree) if, for every parametrically definable path $X$ in $T$ and for every $n \in \mathbb{N}$, there exists $\alpha \in \mathcal{C}$ such that $X \equiv_{n} \alpha$. Equivalently (since the language of trees has finite signature), if every parametrically definable path in $T$ is a model of the first-order theory of $\mathcal{C}$.

If $\mathcal{C}=\{\alpha\}$ then $T$ is simply called an $\alpha$-tree, a uniformly $\alpha$-like tree, etc.

We follow with a few examples that will be used further, in the proof of Theorem 19.

\section{EXAMPLE 13}

The tree $B_{\omega+\mathbf{1}}$ is uncountable. Let $T_{1}$ be any countable elementary substructure of $B_{\omega+\mathbf{1}}$ and let $\mathcal{C}_{1}=\{\omega+\mathbf{1}\} . T_{1}$ is a binary tree not containing any finite paths, so that every path in $T_{1}$ is either an $\omega$-path or an $(\omega+\mathbf{1})$-path. Moreover, $T_{1}$ does actually contain both $\omega$-paths and $(\omega+\mathbf{1})$-paths (and the former are not even 2-equivalent to $\omega+\mathbf{1})$. Thus, $T_{1}$ can be seen as the result of removing an uncountable set of leaves from $B_{\omega+1}$.

Suppose now, that $\varphi(x, \bar{c})$ defines a path $A$ in $\left(T_{1} ; \bar{c}\right)$ for some tuple $\bar{c}$ of nodes from $T_{1}$. Then $\left(T_{1}, \bar{c}\right)=\pi_{\varphi}(\bar{c})$, so $\left(B_{\omega+1}, \bar{c}\right) \models \pi_{\varphi}(\bar{c})$, hence $\varphi(x, \bar{c})$ defines a path in $\left(B_{\omega+1} ; \bar{c}\right)$. Now, since every path in $B_{\omega+1}$ is an $(\omega+\mathbf{1})$-path then we get that $\left(B_{\omega+\mathbf{1}}, \bar{c}\right) \models \exists x(\operatorname{leaf}(x) \wedge \varphi(x, \bar{c}))$ and so $\left(T_{1}, \bar{c}\right) \models \exists x(\operatorname{leaf}(x) \wedge \varphi(x, \bar{c}))$. Hence, $A$ will contain a leaf. Thus, every parametrically definably path in $T_{1}$ will contain a leaf, and since every path in $T_{1}$ containing a leaf is parametrically definable (using that leaf as parameter) it follows that the parametrically definable paths in $T_{1}$ are precisely its $(\omega+\mathbf{1})$-paths.

Thus, $T_{1}$ is a uniformly $(\omega+\mathbf{1})$-like tree, as well as a definably $(\omega+\mathbf{1})$-tree, but neither an $(\omega+\mathbf{1})$-tree, nor a pathwise $(\omega+\mathbf{1})$-like tree. 


\section{EXAMPLE 14}

Let $T_{2}$ be the tree indicated in Figure 1 and let $\mathcal{C}_{2}=\{\omega\}$. Each of the two paths in $T_{2}$ is parametrically definable and elementarily equivalent with $\omega$. In any $\omega$-like tree, every parametrically definable set contains a minimal node. The set of nodes in $T_{2}$ defined by the formula

$$
\varphi(x)=\forall y \forall z(x<y \wedge x<z \rightarrow(y<z \vee y=z \vee z<y))
$$

contains no minimal node. Thus, $T_{2}$ is a definably uniformly $\omega$-like tree, but not $\omega$-like.

Let $\sigma_{1}$ be the sentence

$$
\sigma_{1}:=\exists u \varphi(u) \rightarrow \exists u(\varphi(u) \wedge \forall w(w<u \rightarrow \neg \varphi(w))),
$$

where $\varphi(x)$ is defined as above. This sentence will be used further.

EXAmPLE 15

Let $T_{3}$ be the tree indicated in Figure 1 and let $\mathcal{C}_{3}=\{\mathbf{n}: n \in \mathbb{N}\}$. Both paths in $T_{3}$ are parametrically definable. It is known (e.g. [6]) that for every $m$ there exists some sufficiently large $n$ such that $\omega+\omega^{\star} \equiv_{m} \mathbf{n}$. However, $\omega+\omega^{\star} \not \equiv \mathbf{n}$ for every $n$. In any DU- $\mathcal{C}_{3}$-like tree, the set defined by the formula $\varphi(x)$ from Example 14 will contain a minimal node. However, the subset of $T_{3}$ defined by $\varphi(x)$ does not contain a minimal node. Thus, $T_{3}$ is a definably $\mathcal{C}_{3}$-like tree, even a pathwise $\mathcal{C}_{3}$-like tree, but neither a $\mathcal{C}_{3}$-like tree nor a definably uniformly $\mathcal{C}_{3}$-like tree.

EXAMPLE 16

Let $T_{4}$ be the linear order $\omega+\omega^{\star}$ and let $\mathcal{C}_{4}=\{\mathbf{n}: n \in \mathbb{N}\}$. As noted above, there exists, for every $m$, some sufficiently large $n$ such that $\omega+\omega^{\star} \equiv_{m} \mathbf{n}$, but $\omega+\omega^{\star} \not \equiv \mathbf{n}$ for every $n$.

EXAMPLE 17

Let $T_{5}$ be the tree $B_{\omega}$ and take $\mathcal{C}_{5}=\{\omega+\mathbf{1}\}$. Note that $B_{\omega}$ contains no parametrically definable paths. Let $\sigma_{2}$ be the sentence

$$
\sigma_{2}:=\forall x \exists y(x \leqslant y \wedge \operatorname{leaf}(y))
$$

This sentence will be used further.

EXAMPLE 18

Let $T_{6}$ be the linear order $\omega+\zeta$ and take $\mathcal{C}_{6}=\{\omega\}$. It is known that $\omega \equiv \omega+\zeta$.
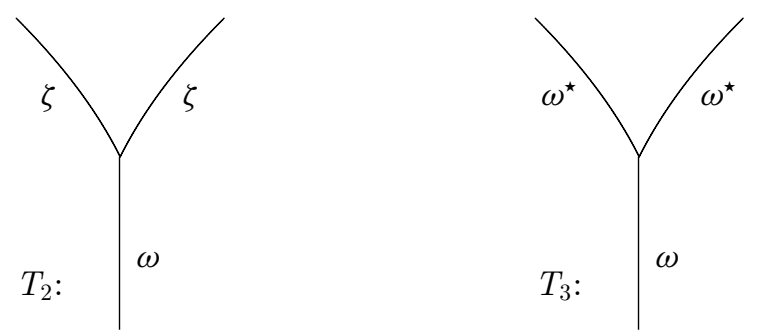

FIg. 1. The trees $T_{2}$ and $T_{3}$ described in Examples 14 and 15. 


\section{Relationships between the $\mathcal{C}$-classes of trees}

THEOREM 19

Let $\mathcal{C}$ be a class of linear orders. The set-theoretical inclusions and non-inclusions that hold between the various $\mathcal{C}$-classes of trees are presented in Figure 2.

Proof. To begin with the inclusions, we will show that the class of $\mathcal{C}$-like trees is contained in the class of D-C-like trees. The argument to show that the class of U-C-like trees is contained in the class of DU-C-like trees is similar. The remaining inclusions are quite easy to verify.

Let $T$ be a $\mathcal{C}$-like tree and let $A$ be a path in $T$ defined in $(T ; \bar{c})$ by the formula $\varphi(x, \bar{c})$ for some tuple of nodes $\bar{c}$ from $T$. Suppose that $A$ has $n$-characteristic $\tau$. Then $T \models \pi_{\varphi}(\bar{c} / \bar{z})$ and $T \models \tau^{\varphi}(\bar{c} / \bar{z})$ so that $T \models \exists \bar{z}\left(\pi_{\varphi}(\bar{z}) \wedge \tau^{\varphi}(\bar{z})\right)$. Since $T$ is $\mathcal{C}$-like then there exists a $\mathcal{C}$-tree $S$ for which $S \models \exists \bar{z}\left(\pi_{\varphi}(\bar{z}) \wedge \tau^{\varphi}(\bar{z})\right)$. Thus, $\varphi(x, \bar{d})$ defines a path $B$ in $(S ; \bar{d})$ for some tuple $\bar{d}$ from $S$, and $B=\tau$. But $B$ is isomorphic with some linear order $C$ in $\mathcal{C}$ and so $A \equiv{ }_{n} C$. It follows that $T$ is a $\mathrm{D}$ - $\mathcal{C}$-like tree.

As an example of a non-inclusion demonstrated by a counterexample, we show that the class of P-C-like trees is not always included in the class of $\mathcal{C}$-like trees. Note, that the tree $T_{2}$ from Example 14 is a $\mathrm{P}-\mathcal{C}_{2}$-like tree, but not a $\mathcal{C}_{2}$-like tree. This is because every $\mathcal{C}_{2}$-like

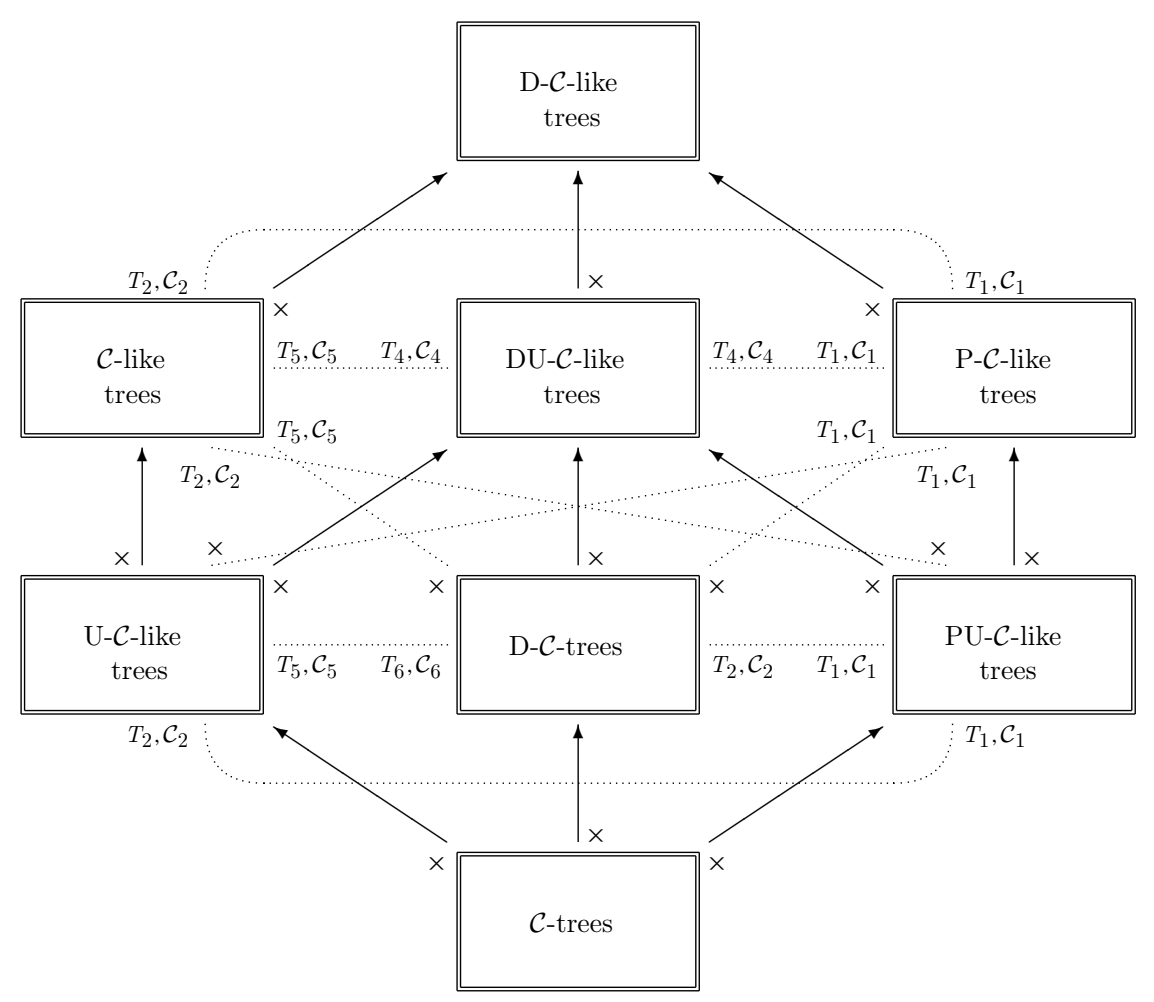

FIG. 2. Relationships between the $\mathcal{C}$-classes of trees (see Theorem 19). Inclusions $X \subseteq Y$ are denoted as $X \rightarrow Y$. Non-inclusions are indicated by specifying a counterexample drawn from Examples 13-18 or, when obtained through completion of diagrams, by $\times$. There are no downwards directed inclusions between classes separated by more than one level. 
tree satisfies the sentence $\sigma_{1}$ as defined in Example 14, while $T_{2}$ does not. Hence, the class of $\mathrm{P}-\mathcal{C}_{2}$-like trees is not contained in the class of $\mathcal{C}_{2}$-like trees.

As an example of a non-inclusion obtained through transitive completion in Figure 2, consider the claim that the class of $\mathrm{P}-\mathcal{C}$-like trees is not generally a subclass of the class of PU-C-like trees. If, to the contrary, the class of $\mathrm{P}-\mathcal{C}$-like trees were a subclass of the class of PU-C-like trees for all classes of linear orders $\mathcal{C}$, then since the class of PU-C-like trees is also a subclass of the class of DU-C-Clike trees for all classes $\mathcal{C}$, we would get that the class of P-C-like trees is a subclass of the class of DU-C-like trees for all classes $\mathcal{C}$. But this contradicts the fact that the tree $T_{4}$ from Example 16 is a $\mathrm{P}-\mathcal{C}_{4}$-like tree, with $\mathcal{C}_{4}$ as defined in Example 16, but $T_{4}$ is not a DU- $\mathcal{C}_{4}$-like tree. This establishes the non-inclusion.

The remaining non-inclusions are easily verified.

The verification of the claims in the following proposition is routine.

Proposition 20

When the class $\mathcal{C}$ consists of a single linear order, the following additional inclusions hold:

(i) the class of P-C-like trees $\subseteq$ the class of PU-C-like trees,

(ii) the class of D-C-like trees $\subseteq$ the class of DU-C-like trees.

Consequently,

(iii) the class of $\mathcal{C}$-like trees $\subseteq$ the class of DU- $\mathcal{C}$-like trees,

(iv) the class of P-C-like trees $\subseteq$ the class of DU-C $\mathcal{C}$-like trees.

\section{Relationships between the first-order theories of $\mathcal{C}$-classes of trees}

THEOREM 21

Let $\mathcal{C}$ be a class of linear orders. The set-theoretical inclusions and non-inclusions that hold between the first-order theories of the various $\mathcal{C}$-classes of trees are presented in Figure 3.

Proof. Let $\sigma \in \mathrm{TH}\left(\mathrm{P}-\mathcal{C}\right.$-like trees) say with $\operatorname{qr}(\sigma)=n$. Let $T$ be a $\mathcal{C}$-like tree. Then $T \equiv_{n} T_{0}$ for some $\mathcal{C}$-tree $T_{0}$. But $T_{0}$ is also a P-C-like tree hence $T_{0} \models \sigma$ and so $T \models \sigma$. It follows that $\sigma \in \mathrm{TH}(\mathcal{C}$-like trees $)$ and so $\mathrm{TH}(\mathrm{P}-\mathcal{C}$-like trees $) \subseteq \mathrm{TH}(\mathcal{C}$-like trees $)$.

The following inclusions can be proven using a similar argument:

- $\mathrm{TH}(\mathrm{DU}-\mathcal{C}$-like trees $) \subseteq \mathrm{TH}(\mathcal{C}$-like trees $)$,

- $\mathrm{TH}(\mathrm{D}-\mathcal{C}$-trees $) \subseteq \mathrm{TH}(\mathcal{C}$-like trees $)$,

- $\mathrm{TH}(\mathrm{PU}-\mathcal{C}$-like trees $) \subseteq \mathrm{TH}(\mathcal{C}$-like trees $)$,

- $\mathrm{TH}(\mathrm{U}-\mathcal{C}$-like trees $) \subseteq \mathrm{TH}(\mathcal{C}$-like trees $)$,

- $\mathrm{TH}(\mathrm{PU}-\mathcal{C}$-like trees $) \subseteq \mathrm{TH}(\mathrm{U}-\mathcal{C}$-like trees $)$,

- $\mathrm{TH}(\mathrm{P}-\mathcal{C}$-like trees $) \subseteq \mathrm{TH}(\mathrm{U}-\mathcal{C}$-like trees $)$,

- $\mathrm{TH}(\mathrm{D}-\mathcal{C}$-trees $) \subseteq \mathrm{TH}(\mathrm{U}-\mathcal{C}$-like trees $)$.

The inclusion $\mathrm{TH}(\mathcal{C}$-trees $) \subseteq \mathrm{TH}(\mathrm{U}-\mathcal{C}$-like trees $)$ is immediate.

The remaining inclusions follow from Theorem 19 and the accompanying diagram in Figure 2 .

We briefly discuss the non-inclusions shown in Figure 3. Consider, for example, the non-inclusion $\mathrm{TH}\left(\mathrm{DU}-\mathcal{C}_{3}\right.$-like trees $) \nsubseteq \mathrm{TH}\left(\mathrm{P}-\mathcal{C}_{3}\right.$-like trees $)$. To see this, let $T$ be a definably 


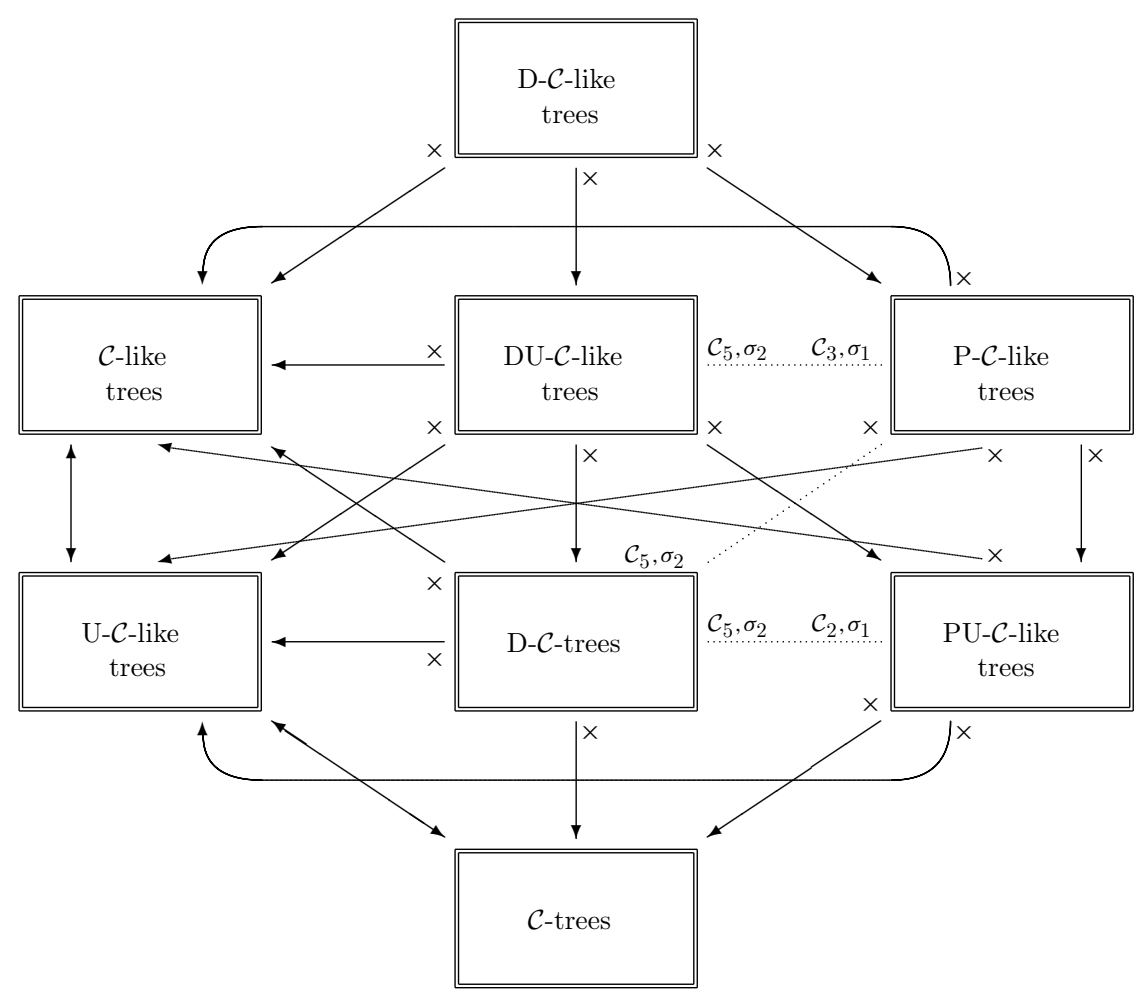

FIG. 3. Relationships between the first-order theories of the $\mathcal{C}$-classes of trees (see Theorem 21). Inclusions $X \subseteq Y$ are denoted as $X \rightarrow Y$. Non-inclusions are indicated by specifying a counterexample drawn from Examples 13-18 or, when obtained through completion, by the symbol $\times$. Except for the obvious cases, there are no upwards directed inclusions between the theories of classes separated by more than one level.

uniformly $\mathcal{C}_{3}$-like tree and suppose that $T \models \varphi(a / x)$ for some $a \in T$, where $\varphi(x)$ is as in Example 14. Then $a$ belongs to a parametrically definable (being 'singular', see next section) path $A$, with $A \equiv \mathbf{n}$ for some $n \in \mathbb{N}$. Hence, $A$ will be finite. It follows that $T \models \sigma_{1}$ (where $\sigma_{1}$ is as in Example 14) and so $\sigma_{1} \in \mathrm{TH}\left(\mathrm{DU}-\mathcal{C}_{3}\right.$-trees). However, $T_{3}$ is a P- $\mathcal{C}_{3}$-like tree with $T_{3} \not \sigma_{1}$, so that $\sigma_{1} \notin \mathrm{TH}\left(\mathrm{P}-\mathcal{C}_{3}\right.$-like trees $)$. This serves as a counterexample to establish the non-inclusion $\mathrm{TH}\left(\mathrm{DU}-\mathcal{C}_{3}\right.$-like trees $) \nsubseteq \mathrm{TH}\left(\mathrm{P}-\mathcal{C}_{3}\right.$-like trees $)$.

It can be shown, using an argument similar to the one in the preceding paragraph, that $\mathrm{TH}\left(\mathrm{D}-\mathcal{C}_{2}\right.$-trees $) \nsubseteq \mathrm{TH}\left(\mathrm{PU}-\mathcal{C}_{2}\right.$-like trees $)$.

The non-inclusions which use the class $\mathcal{C}_{5}$ and the sentence $\sigma_{2}$ from Example 17 as counterexample are easily verified.

Finally, the non-inclusions obtained through completion are trivial. For example, $\mathrm{TH}(\mathcal{C}$-trees $)$ is not generally a subtheory of $\mathrm{TH}(\mathrm{D}-\mathcal{C}$-trees $)$, for if it were, then using the fact that $\mathrm{TH}(\mathrm{PU}-\mathcal{C}$-like trees $) \subseteq \mathrm{TH}(\mathcal{C}$-trees $)$ for all classes $\mathcal{C}$, this would give $\mathrm{TH}(\mathrm{PU}-\mathcal{C}$-like trees $) \subseteq \mathrm{TH}(\mathrm{D}-\mathcal{C}$-trees $)$ for all classes $\mathcal{C}$, contradicting the fact that $\mathrm{TH}\left(\mathrm{PU}-\mathcal{C}_{5}\right.$-like trees $) \nsubseteq \mathrm{TH}\left(\mathrm{D}-\mathcal{C}_{5}\right.$-trees $)$. 
Likewise the theory $\mathrm{TH}(\mathcal{C}$-like trees $)$ is not generally a subtheory of the theory $\mathrm{TH}(\mathrm{D}$ - $\mathcal{C}$-trees $)$, for if it were, then the theories $\mathrm{TH}(\mathrm{D}-\mathcal{C}$-trees $), \mathrm{TH}(\mathcal{C}$-like trees $)$ and $\mathrm{TH}(\mathrm{U}-\mathcal{C}$-like trees) would coincide for all classes $\mathcal{C}$. But this would contradict the fact that $\mathrm{TH}(\mathcal{C}$-trees $) \subseteq \mathrm{TH}(\mathrm{U}-\mathcal{C}$-like trees $)$ for all classes $\mathcal{C}$, while there exist classes $\mathcal{C}$ for which $\mathrm{TH}(\mathcal{C}$-trees $) \nsubseteq \mathrm{TH}(\mathrm{D}-\mathcal{C}$-trees $)$.

The remaining non-inclusions can be proved using similar reasoning.

Proposition 22

If $\mathcal{C}$ consists of a single linear order, the following additional inclusions hold between the first-order theories of $\mathcal{C}$-classes of trees:

(i) $\mathrm{TH}(\mathrm{DU}-\mathcal{C}$-like trees $) \subseteq \mathrm{TH}(\mathrm{D}-\mathcal{C}$-like trees $)$,

(ii) $\mathrm{TH}(\mathrm{DU}-\mathcal{C}$-like trees $) \subseteq \mathrm{TH}(\mathrm{P}-\mathcal{C}$-like trees $)$,

(iii) $\mathrm{TH}(\mathrm{PU}-\mathcal{C}$-like trees $) \subseteq \mathrm{TH}(\mathrm{P}-\mathcal{C}$-like trees $)$.

The other non-inclusions remain the same.

\section{Axiomatizing the first-order theories of the $\mathcal{C}$-classes of trees}

As shown in the previous section, every class of linear orders generates eight naturally associated classes of trees, which in general have up to six different first-order theories. In this section, we discuss the following problem: given a class of linear orders $\mathcal{C}$, can we determine the first-order theories of the respective $\mathcal{C}$-classes of trees in terms of the firstorder theory of $\mathcal{C}$ ? In principle, this is a rather difficult problem, because $\mathcal{C}$ may consist of a quite arbitrary collection of linear order types. Here we will obtain some general results, for some of the associated classes of trees; more specific results will be included in a follow-up work.

A natural family of axioms for the class of trees constructed from $\mathcal{C}$ arises as follows: every parametrically definable path in every such tree must satisfy all axioms of the theory of $\mathcal{C}$. To be more precise, for any theory of linear orders $\Sigma$, let $D e_{\Sigma}$ denote the scheme consisting of all sentences

$$
\forall \bar{z}\left(\pi_{\varphi}(\bar{z}) \rightarrow \sigma^{\varphi}(\bar{z})\right)
$$

for every formula $\varphi(x, \bar{z})$ (including formulas $\varphi(x)$ for which the tuple $\bar{z}$ is empty) and for every sentence $\sigma \in \Sigma$. If $\Sigma=\{\sigma\}$ then $\mathrm{De}_{\Sigma}$ is written simply as $\mathrm{De}_{\sigma}$. The scheme $\mathrm{De}_{\Sigma}$ states that every parametrically definable path satisfies the theory $\Sigma$.

This axiom scheme axiomatizes the class of definably $\mathcal{C}$-like trees:

Proposition 23

Let $\mathcal{C}$ be a class of linear order types axiomatized by the theory $\Sigma$. The class of definably $\mathcal{C}$-like trees is precisely the class of models of the theory Tree $\cup D e_{\Sigma}$.

Proof. Let $T$ be a definably $\mathcal{C}$-like tree. It is immediate that $T$ satisfies Tree. Let $\varphi(x, \bar{z})$ be a formula with $\bar{z}=\left(z_{1}, \ldots, z_{k}\right)$ ( $\bar{z}$ may be empty), let $\bar{c}=\left(c_{1}, \ldots, c_{k}\right)$ be a tuple of nodes from $T$, and let $T \models \pi_{\varphi}(\bar{c} / \bar{z})$. Then there is a path $A$ defined in $(T ; \bar{c})$ by $\varphi(x, \bar{c})$. But $A=\sigma$ for every $\sigma \in \Sigma$ and $A=(T ; \bar{c})^{\varphi}$, so $T \models \sigma^{\varphi}(\bar{c} / \bar{z})$ for every $\sigma \in \Sigma$. Thus, $T \models \operatorname{De}_{\Sigma}$.

Conversely, let $T$ be a structure such that $T=\operatorname{Tree} \cup D e_{\Sigma}$. Then $T$ is a tree. Let $\bar{c}$ be a (possibly empty) $k$-tuple of nodes in $T$, let $\varphi(x, \bar{z})$ be a formula with $\bar{z}=\left(z_{1}, \ldots, z_{k}\right)$, and let $A$ be a path definable in $(T ; \bar{c})$ using the formula $\varphi(x, \bar{c})$. Then $T=\pi_{\varphi}(\bar{c} / \bar{z})$ hence $T \models \sigma^{\varphi}(\bar{c} / \bar{z})$ 
for every $\sigma \in \Sigma$. But $A=(T ; \bar{c})^{\varphi}$ so $A=\sigma$ for every $\sigma \in \Sigma$. Hence, $A$ is a model of $\mathrm{TH}(\mathcal{C})$. Thus, $T$ is a definably $\mathcal{C}$-like tree.

When $\mathcal{C}$ is an axiomatizable class of linear order types, the classes of definably $\mathcal{C}$-like trees and definably $\mathcal{C}$-trees coincide, hence the following holds.

Corollary 24

Let $\mathcal{C}$ be an axiomatizable class of linear order types, axiomatized by the theory $\Sigma$. Then the class of definably $\mathcal{C}$-trees is precisely the class of models of the theory Tree $\cup D e_{\Sigma}$.

Likewise, if $\mathcal{C}$ consists of finitely many linear order types, then the classes of definably $\mathcal{C}$-like trees and definably uniformly $\mathcal{C}$-like trees coincide, hence the following holds.

Corollary 25

Let $\mathcal{C}$ be a finite class of linear order types, axiomatized by the theory $\Sigma$. Then the class of definably uniformly $\mathcal{C}$-like trees is precisely the class of models of the theory Tree $\cup D e_{\Sigma}$.

As is well known (see e.g. [6]), for every ordinal $\alpha$ with $\alpha<\omega^{\omega}$, there exists a first-order sentence $\Phi_{\alpha}$ which axiomatizes the first-order theory of $\alpha$, and $\Phi_{\alpha} \equiv \Phi_{\beta}$ if and only if $\alpha=\beta$. Hence, we have the following corollary.

Corollary 26

Let $\alpha$ be an ordinal with $\alpha<\omega^{\omega}$. The class of definably (uniformly) $\alpha$-like trees is precisely the class of models of the theory Tree $\cup D e_{\Phi_{\alpha}}$.

Now, what about the other $\mathcal{C}$-classes of trees, characterized in terms of the entire structures, or the sets of all paths in them, not only the parametrically definable ones? To get a grip on the axiomatizations of such classes one has to analyse the non-definable paths emerging in them. We will consider that question in the next section, where we will take a closer look at first-order parametrically definable and undefinable paths in trees.

\section{First-order parametrically definable and undefinable paths in trees: zooming in}

\subsection{On parametrically definable paths}

For $\alpha$ an ordinal and $T$ a well-founded tree, a node $a$ in $T$ is said to have level $\alpha$ when $\alpha$ is the order type of the set $a_{>}$. The supremum of the set of all levels of nodes in $T$ is called the height of $T$. The set of nodes having level $\alpha$ can be defined using the formula

$$
\operatorname{level}_{\alpha}(y):=\Phi_{\alpha}^{\theta(x, y)}(y)
$$

where $\theta(x, y):=x \leqslant y$.

The next result shows that in well-founded trees $T$ of height less than $\omega^{\omega}$, the ability of nodes to define subsets (in particular, paths) of $T$, improves with the level of those nodes.

Proposition 27

Let $T$ be a well-founded tree of height less than $\omega^{\omega}$. Let $\bar{c}=\left(c_{1}, \ldots, c_{k}\right)$ be a tuple of nodes from $T$ and let $\varphi(x, \bar{z})$ be a formula, with $\bar{z}=\left(z_{1}, \ldots, z_{k}\right)$, such that $\varphi(x, \bar{c})$ defines the set $A$ in $(T ; \bar{c})$. For every $i(1 \leqslant i \leqslant k)$, let $d_{i} \in T$ with $c_{i} \leqslant d_{i}$. Then there is a formula $\psi(x, \bar{z})$ such that $\psi(x, \bar{d})$ defines $A$ in $(T ; \bar{d})$, where $\bar{d}=\left(d_{1}, \ldots, d_{k}\right)$. 
Proof. For every $i$, suppose $c_{i}$ has level $\alpha_{i}$. Then $c_{i}$ can be defined in $(T ; \bar{d})$ using the formula $\gamma_{i}\left(y, d_{i}\right):=y \leqslant d_{i} \wedge \operatorname{level}_{\alpha_{i}}(y)$. Hence take

$$
\psi\left(x, z_{1}, \ldots, z_{k}\right):=\forall y_{1} \ldots \forall y_{k}\left(\bigwedge_{i=1}^{k} \gamma_{i}\left(y_{i}, z_{i}\right) \rightarrow \varphi\left(x, y_{1}, \ldots, y_{k}\right)\right) .
$$

In particular, in well-founded trees of height less than $\omega^{\omega}$ for which every non-leaf node has a successor which is a leaf, every parametrically definable set of nodes can be defined using leaves for parameters.

Proposition 28

Let $T$ be a finitely branching tree in which every node has finite level and let $A$ be a path in $T$. Suppose $A$ is definable in $(T ; \bar{c})$ for some tuple of nodes $\bar{c}=\left(c_{1}, \ldots, c_{k}\right)$ from $T$. Then there exists $d \in A$ such that $A$ is definable in $(T ; d)$.

Proof. We first show that the parameter $c_{k}$ can be replaced with a parameter $d_{k}$ from $A$ itself. Let $\varphi(x, \bar{z})$ be a formula (with $\left.\bar{z}=\left(z_{1}, \ldots, z_{k}\right)\right)$ such that $\varphi(x, \bar{c})$ defines $A$ in $(T ; \bar{c})$. Suppose $\varphi$ has quantifier rank $n$ and that $c_{k}$ has level $l$. Let

$$
B:=\left\{b \in T: T \models \llbracket(T ; \bar{c}) \rrbracket^{n+2}\left(c_{1} / x_{1}, \ldots, c_{k-1} / x_{k-1}, b / x_{k}\right)\right.
$$

and $b$ has level $l\}$.

Since $c_{k} \in B$ we have that $B$ is non-empty. From the fact that $T$ is finitely branching and that every node in $T$ has finite level it follows that $B$ is finite. $B$ can be defined in $\left(T ; c_{1}, \ldots, c_{k-1}\right)$ using the formula $\xi\left(x, c_{1}, \ldots, c_{k-1}\right)$, where

$$
\xi\left(x, z_{1}, \ldots, z_{k-1}\right):=\llbracket(T ; \bar{c}) \rrbracket^{n+2}\left(z_{1}, \ldots, z_{k-1}, x\right) \wedge \operatorname{level}_{l}(x) .
$$

Since $(T ; \bar{c}) \models \pi_{\varphi}(\bar{c})$ and $\left(T ; c_{1}, \ldots, c_{k}\right) \equiv_{n+2}\left(T ; c_{1}, \ldots, c_{k-1}, b\right)$ for every $b \in B$ then $\varphi\left(x, c_{1}, \ldots, c_{k-1}, b\right)$ defines a path in $\left(T ; c_{1}, \ldots, c_{k-1}, b\right)$ for every $b \in B$. Hence, the formula $\zeta\left(x, c_{1}, \ldots, c_{k-1}\right)$, where

$$
\zeta\left(x, z_{1}, \ldots, z_{k-1}\right):=\exists y\left(\xi\left(y, z_{1}, \ldots, z_{k-1}\right) \wedge \varphi\left(x, z_{1}, \ldots, z_{k-1}, y\right)\right)
$$

defines in $\left(T ; c_{1}, \ldots, c_{k-1}\right)$ a subtree $T_{0}$ of $T$ containing only finitely many paths, amongst which is $A$.

Now, choose any $d_{k} \in A$ such that $d_{k}$ does not belong to any path in $T_{0}$ except for $A$. Then $A$ can be defined in $\left(T ; c_{1}, \ldots, c_{k-1}, d_{k}\right)$ using the formula $\chi\left(x, c_{1}, \ldots, c_{k-1}, d_{k}\right)$, where

$$
\chi\left(x, z_{1}, \ldots, z_{k}\right):=\zeta\left(x, z_{1}, \ldots, z_{k-1}\right) \wedge\left(x<z_{k} \vee x=z_{k} \vee z_{k}<x\right)
$$

Hence, we have succeeded in replacing the parameter $c_{k}$ with a parameter $d_{k}$ from $A$.

Repeating this procedure for the parameters $c_{k-1}, \ldots, c_{1}$, we eventually obtain nodes $d_{1}, \ldots, d_{k} \in A$ and a formula $\chi^{\prime}\left(x, z_{1}, \ldots, z_{k}\right)$ such that $\chi^{\prime}\left(x, d_{1}, \ldots, d_{k}\right)$ defines $A$ in $\left(T ; d_{1}, \ldots, d_{k}\right)$. Suppose without loss of generality that $d_{i} \leqslant d_{1}$ for every $i \geqslant 2$ and that the level of $d_{i}$ is $l_{i}$. 
Then $d_{i}$ can be defined in $\left(T ; d_{1}\right)$ using the formula $x \leqslant d_{1} \wedge \operatorname{level}_{l_{i}}(x)$. It follows that $A$ can be defined in $\left(T ; d_{1}\right)$ using the formula $\psi\left(x, d_{1}\right)$, where

$$
\psi(x, z):=\forall z_{2} \ldots \forall z_{k}\left(\bigwedge_{i=2}^{k}\left(z_{i} \leqslant z \wedge \operatorname{level}_{l_{i}}\left(z_{i}\right)\right) \rightarrow \chi^{\prime}\left(x, z, z_{2}, \ldots, z_{k}\right)\right) .
$$

Now, take $d=d_{1}$.

\subsection{Singular and emergent paths}

It is generally not possible to formalize in the first-order language of trees with equality the requirement that all paths in a given tree satisfy the first-order theory of some class $\mathcal{C}$ of linear orders, and subsequently to axiomatize the first-order theory of the class of $\mathcal{C}$-trees. This is because not all paths in a tree need to be parametrically definable, so we need to analyse deeper the nature of non-parametrically definable paths in trees, too. The behaviour and structure of such paths can be better understood by considering two types of paths: singular and emergent.

\section{DeFINITION 29}

Let $T$ be a tree and let $A$ be a path in $T$. $A$ is called singular if there exists $a \in A$ such that $a_{\leqslant}$is total. Otherwise the path $A$ is called emergent. If $\mathcal{B}$ is a set of paths from $T$ with $A \notin \mathcal{B}$ and with $A \subseteq \bigcup \mathcal{B}$ then $A$ is said to emerge from $\mathcal{B}$.

For a more detailed analysis of singular and emergent paths, the reader is referred to [5].

EXAMPLE 30

Let $T$ be the tree obtained by taking the linear order $A:=\omega$ and at each point in $A$, we adjoin a copy of $\omega$ (see Figure 4). Thus, every path in $T$ will be isomorphic with $\omega$. The path $A$ is an emergent path, while every other path in $T$ is singular.

Clearly, every path containing a greatest node is singular.

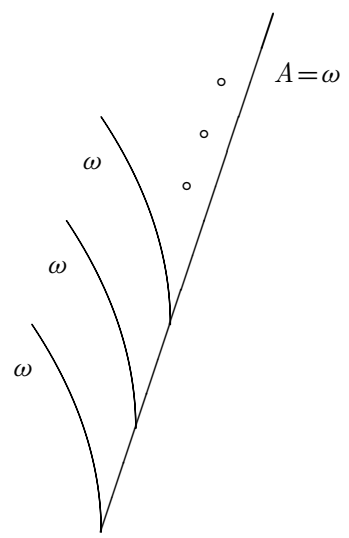

FIG. 4. Singular and emergent paths (see Example 30). 
Every singular path is parametrically definable. Indeed, let $A$ be a singular path in a tree $T$ and let $a \in A$ such that $a_{\leqslant}$is total. Then $A$ can be defined in $(T ; a)$ using the formula

$$
x<a \vee x=a \vee a<x .
$$

It hence follows that, given any tree $T$, the set of paths in $T$ which are not parametrically definable forms a subset of the set of emergent paths.

Note, however, that not all emergent paths need be undefinable. For example, the path $A$ in the tree $T$ from Example 30 is definable in $T$ using the formula

$$
\exists y_{1} \exists y_{2}\left(y_{1} \neq y_{2} \wedge \bigwedge_{i=1}^{2}\left(x<y_{i}\right) \wedge \bigwedge_{i=1}^{2}\left(\neg \exists z\left(x<z \wedge z<y_{i}\right)\right)\right)
$$

\subsection{On non-definable paths}

First, we will show that if a path $A$ in a tree $T$ is not parametrically definable, then there exists a 'ladder' of paths in $T$, different from $A$, such that $A$ cannot be distinguished from the path corresponding to the $n$-th step of the 'ladder' by using first-order formulae of quantifier rank $n$.

\section{LEMMA 31}

Let $T$ be a tree and let $A$ be a path that is not parametrically definable in $T$. For every $a \in A$ and for every $n \in \mathbb{N}$, there exists $b \in A$ and $c \in T \backslash A$ with $b, c \geqslant a$ and such that $b_{\leqslant} \equiv_{n} c_{\leqslant}$.

Proof. Let $a \in A$ and let $n \in \mathbb{N}$ but suppose to the contrary that $x_{\leqslant} \neq_{n} y \leqslant$ for every $x \in A$ and for every $y \in T \backslash A$ with $x, y \geqslant a$. Let $\tau_{1}, \ldots, \tau_{m}$ be all $n$-characteristics of empty tuples over the language of ordered sets. Let $U=\left\{i: x_{\leqslant} \models \tau_{i}\right.$ for some $x \in A$ with $\left.x \geqslant a\right\}$. Then for every $x$ satisfying $x \geqslant a$, we have that $x \leqslant \models \tau_{i}$ for some $i \in U$ if and only if $x \in A$. Let $\theta(u, y)=y \leqslant u$. Then $A$ can be defined in $(T ; a)$ using the formula

$$
\varphi(x, a):=x<a \vee x \geqslant a \wedge\left(\bigvee_{i \in U} \tau_{i}^{\theta(u, a)}\right),
$$

a contradiction.

Proposition 32

Let $\alpha$ be a linear order containing a greatest element and suppose the first-order theory of $\alpha$ can be axiomatized using the sentence $\sigma$. Let $T$ be a definably (uniformly) $\alpha$-like tree containing only finitely many paths which are not parametrically definable. Then for every $n \in \mathbb{N}$, there exists a pathwise uniformly $\alpha$-like tree $S$ such that $S \equiv_{n} T$.

Proof. It suffices to prove the result for large $n$, so let $n \geqslant \operatorname{qr}(\sigma)+1$. Let $A_{1}, \ldots, A_{k}$ be the paths in $T$ that are not parametrically definable and for every $i$, let $a_{i} \in A_{i}$ be such that $a_{i} \notin A_{j}$ for all $j(j \neq i)$. By Lemma 31, for every $i$, there exists $b_{i} \in A_{i}$ and $c_{i} \in T \backslash A_{i}$ with $b_{i}, c_{i} \geqslant a_{i}$ and such that $\left(b_{i}\right)_{\leqslant} \equiv_{n}\left(c_{i}\right) \leqslant$.

Let $S$ be the tree obtained by taking the tree $T$ and, for every $i$, replacing the subtree $\left(b_{i}\right) \leqslant$ with the tree $S_{i}:=\left(c_{i}\right)_{\leqslant}$. From the way $S$ is constructed, every path in $S$ will contain a leaf node and hence every path in $S$ is definable using that leaf as parameter. Let $\theta(x, y):=x \leqslant y$ 
and define $\tau:=\forall y\left(\operatorname{leaf}(y) \rightarrow \sigma^{\theta(x, y)}(y)\right)$. Note that $\operatorname{qr}(\tau)=\operatorname{qr}(\sigma)+1$. By Proposition 11, we get $T \equiv_{n} S$ and since $T$ satisfies $\tau$ then $S$ also satisfies $\tau$. Since every path in $S$ contains a leaf then it follows that every path in $S$ satisfies $\sigma$ and hence $S$ is pathwise uniformly $\alpha$-like.

\section{Concluding remarks}

In this article, we have started a systematic study of the classes and first-order theories of trees, naturally associated, in terms of the paths occurring in those trees and the firstorder theories of those paths, with a given class $\mathcal{C}$ of linear orders. We have identified eight generally different $\mathcal{C}$-classes of trees and have classified their set-theoretic relationships and the respective relationships between their first-order theories in the general case, i.e. without any specific assumptions about the $\operatorname{class} \mathcal{C}$. We have then obtained some general results about the axiomatizations of the first-order theories of some $\mathcal{C}$-classes of trees and have indicated the problems obstructing such general results for the other classes. These problems arise from the possible emergence of non-definable paths in the trees, that need not satisfy the first-order theory of $\mathcal{C}$, so we have started analysing definable and undefinable paths in trees. In a follow-up paper, we will apply these, and further results, to establish complete axiomatizations of the first-order theories of the classes of $\mathcal{C}$-trees for some important classes $\mathcal{C}$ of linear orders.

\section{Funding}

This work was supported by the Department of Labour of South Africa and the National Research Foundation of South Africa.

\section{References}

[1] R. Backofen, J. Rogers, and K. Vijay-Shankar. A first-order axiomatization of the theory of finite trees. Journal of Logic, Language and Information, 4, 5-39, 1995.

[2] K. Doets. Completeness and definability: applications of the Ehrenfeucht game in second-order and intensional logic. PhD Thesis, University of Amsterdam, 1987.

[3] H.-D. Ebbinghaus, J. Flum, and W. Thomas. Mathematical Logic. Springer, 1996.

[4] V. Goranko. Trees and finite branching. In Proceedings of the 2nd Panhellenic Logic Symposium, July 13-17, 1999, Delphi, Greece. P. Kolaitis and G. Koletsos, eds, pp. 91-101. National Technical University of Athens, 1999.

[5] V. Goranko and A. Zanardo. From linear to branching-time temporal logics: transfer of semantics and definability. Logic Journal of the IGPL, 15, 53-76, 2007.

[6] J. Rosenstein. Linear Orderings. Academic Press, 1982.

[7] J. Schmerl. On $\aleph_{0}$-categoricity and the theory of trees. Fundamenta Mathematicae, 94, 121-128, 1977.

Received 25 April 2010 